\title{
Creation of recombination corrective algorithm for research of a wind tunnel parameters
}

Cite as: AIP Conference Proceedings 2118, 030050 (2019); https://doi.org/10.1063/1.5114778 Published Online: 27 June 2019

Vitalii Yanovych, Daniel Duda, Vít Horáček, and Václav Uruba

\section{ARTICLES YOU MAY BE INTERESTED IN}

Sciences Committee and Reviewers: 38th Meeting of Departments of Fluid Mechanics and Thermodynamics

AIP Conference Proceedings 2118, 010002 (2019); https://doi.org/10.1063/1.5114725

Influence of secondary flow corner vortex to boundary layer in a channel flow AIP Conference Proceedings 2118, 030011 (2019); https://doi.org/10.1063/1.5114739

History of simulation of transient temperature fields of solidifying metals with phase change AIP Conference Proceedings 2118, 020001 (2019); https://doi.org/10.1063/1.5114726

\section{Conference Proceedings}

Get $30 \%$ off all print proceedings!

\section{Enter Promotion Code PDF30 at checkout}

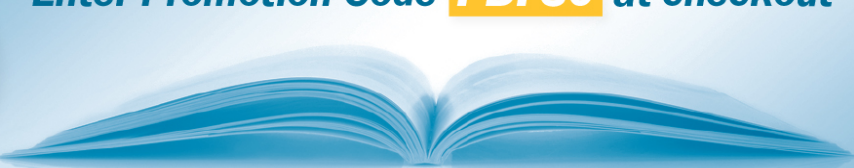




\title{
Creation of Recombination Corrective Algorithm for Research of a Wind Tunnel Parameters
}

\author{
Vitalii Yanovych ${ }^{1, a)}$, Daniel Duda ${ }^{1, c)}$, Vít Horáček ${ }^{1, \text { d) }}$ and Václav Uruba ${ }^{1,2, b)}$ \\ ${ }^{1}$ Faculty of Mechanical Engineering, University of West Bohemia in Pilsen,Univerzitni 22, 30614 Pilsen, \\ Czech Republic \\ ${ }^{2}$ Institute of Thermodynamics, Czech Academy of Sciences,Dolejškova 5, 182 00, Prague, Czech Republic. \\ a)Corresponding author: yanovichvitaliy@i.ua \\ b)uruba@kke.zcu.cz \\ c)dudad@kke.zcu.cz \\ d)vhoracek@students.zcu.cz
}

\begin{abstract}
The blown down wind tunnel tube has been developed. It provides a high degree of air flow laminarization. The uniqueness of this design is the presence of a system of air vortices destruction. This system contains a complex of metal grids, honeycomb straightener and contraction nozzle. The combination of these elements allows for low turbulence level and promote the air flow relative to its axis. The complex of measuring equipment for the establishment of operational characteristics in the test section has been performed. A four-channel probe has been developed to analyze the total pressure and air flow velocity in the measuring tunnel area. The design of this probe makes possible to analyze the boundary layers of air flow in a wind tunnel. The corrective algorithm of recombination for the obtained data and software for their analysis is developed. A prerequisite for developing an algorithm for the recombination of the obtained data and their subsequent visualization is the idea of sectoral analysis and averaging of the data of common areas of the measuring area. Besides the developed algorithm, the correction function for leveling the aerodynamic resistance of the design of the four-channel probe was included. Whereas it approaches the nozzle of static pressure, there is a distortion of the real data distribution on each of the probe tube. To confirm the adequacy of the developed algorithm and the correct functioning of the software developed on its basis, a series of experimental studies was conducted, which were based on the measurement of total pressure when placing objects that create aerodynamic resistance in the measuring section. A profile analysis of the data allowed to visualize correctly the distribution of total pressure around the investigated bodies, even in the place of its sharp difference.
\end{abstract}

\section{INTRODUCTION}

The study of the power interaction of the flow and the physical body is one of the most important tasks of aerodynamics, which is solved by experiment in wind tunnels. In most cases, the principle of reverse is used when the model is fixed in the test section, and the flow of gas enters it at a given speed [1-4]. This principle, in accordance with the general principle of the relativity of classical mechanics, is used in the case of homogeneous linear rectilinear movement of the body [5-7]. Basically all currently used wind tunnels can be divided by structural features into close- and open-circuit types. The modern aerodynamic experiment provides a large range of measurements of the flow parameters of the gas flowing around the model [8-11]. The main types of measurements in wind tunnels are related to the study of the flow around physical bodies and the definition of their aerodynamic characteristics [12]. The other measurements are related to the definition of the wind stream parameters. In this case, the measurement of pressure and velocity, as well as the definition of the velocity field in the working part of the wind tunnel, are of great importance [13, 14]. 


\section{Development of Laminar Wind Tunnel and Description Auxiliary Equipment}

In the laboratory of the Department of Power Machines University of West Bohemia in Plzen (Czech Republic) open-circuit wind tunnel was designed and built. The tunnel could be characterized by the high degree of laminar air flow, which allows to carry out with high accuracy the research of aerodynamic characteristics of complex bodies and turbulent streams created by them.

In collaboration with WTTech.CZ, the 3D model of the wind tunnel was developed using the Autodesk Inventor software environment (Fig. 1a). On the basis of the developed 3D model, a pilot probe was prepared together with traversing system and auxiliary equipment (Fig. 1b).

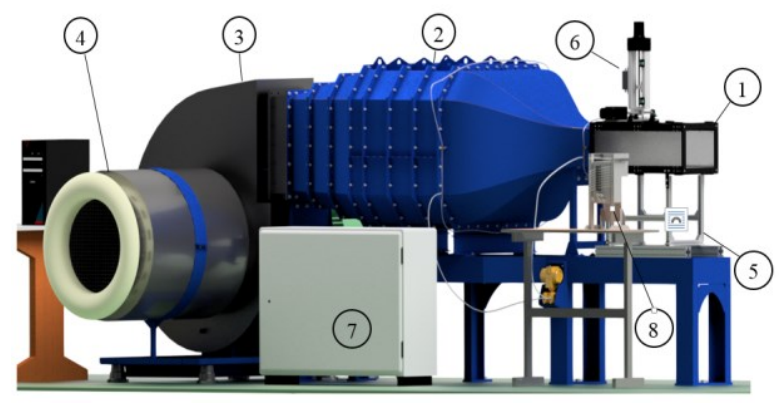

(a)

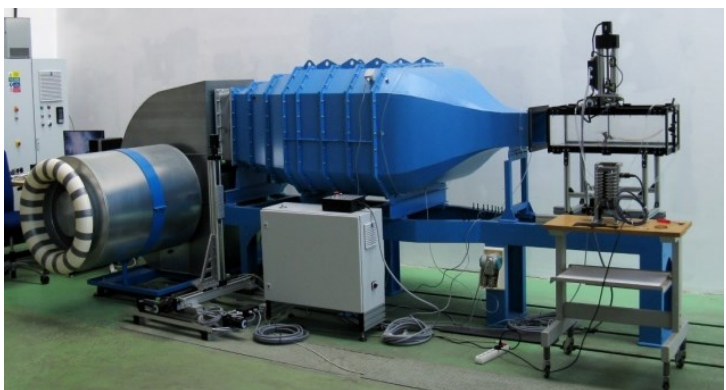

(b)

FIGURE 1. Experimental model of the wind tunnel and equipment for its research. (a) 3D model of wind tunnel, (b) implementation of the tunnel, (1) test section, (2) a module for air flow laminarization, (3) ventilator, (4) air filter, (5) frame, (6) $2 \mathrm{D}$ traverse, (7) the switching block of the traverse, (8) pressure analysis system.

The developed wind tunnel design relates to low-noise tunnels. It allows you to create an airflow rate of up to $80 \mathrm{~m} \cdot \mathrm{s}^{-1}$ which is significantly lower than the speed of sound. Under these conditions, the resulting flow can be considered incompressible.

The uniqueness of the developed wind tunnel lies in its ability to create high-laminar air flow. That became possible due to the use of the laminarization module. This module contains diffuser, settling chamber for laminarization air flow, as well as an output contraction nozzle. The main purpose of the developed wind tunnel is to destroy the turbulence present in the air flow.

To determine the profile of total pressure and flow velocity in the measuring plane, a special probe with four Pitot tubes was developed (Fig. 2). Probe tubes allow for measurement of total pressure. Whereas the static pressure required to calculate the dynamic pressure and flow velocity is measured by a separate tube located at the top of the measuring chamber. The outer diameter of the tube is $2 \mathrm{~mm}$, the inner diameter is $1.6 \mathrm{~mm}$, the length is $55 \mathrm{~mm}$. The peculiarity of the design of the developed probe allows a high accuracy to measure the boundary layer of the air flow. The movement of the probe relative to the measuring plane is carried out by the movement of the traverse, which in turn is secured by the probe.

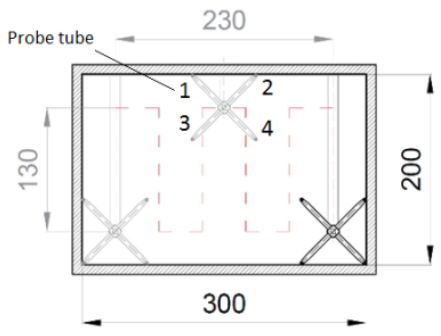

(a)

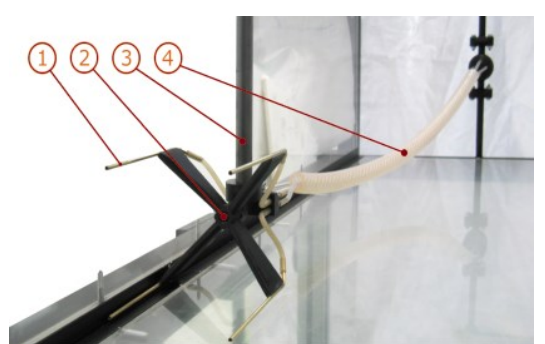

(b)

FIGURE 2. Scheme of an experimental model of a four-chanel probe. (a) To the left there is the trajectory of the probe movement relative to the measuring area. (b) The design of the probe from the switching system of the branch pipes is shown on the right. (1) Pito tube, (2) probe frame, (3) traverse, (4) pipes for supplying pressure to the measuring equipment. 
The presence of a four-channel probe and its complex trajectory of motion in the measuring section of the developed wind tunnel does not allow the use of a classical linear algorithm for the recombination of the data obtained. This tendency is due to the eccentric arrangement of the measuring nozzles relative to the central axis of the probe by $35 \mathrm{~mm}$. Thus, the actual measurement coordinates, and as a consequence, the data obtained for each of the nozzles are displaced relative to the measuring plane by $70 \mathrm{~mm}$. In this case, the range of the traverse is $\mathrm{OX}=0-230 \mathrm{~mm}, \mathrm{OY}=0-130 \mathrm{~mm}$.

\section{Description Recombination Corrective Algorithm}

A prerequisite for developing an algorithm for the recombination of the obtained data and their subsequent visualization is the idea of sectoral analysis and averaging of the data of common areas of the measuring area. Based on the above idea, a series of experimental measurements of the dynamic pressure in the measuring area of the wind tunnel was conducted. The graphic interpretation of the data obtained showed an uneven distribution of data with a series of maximum and minimum deviations. This phenomenon is due to the presence of some aerodynamic resistance to the design of a four-channel probe. Which, when approaching the nozzle of static pressure, causes distortion of the real data distribution on each of the branch pipes. Therefore, for further recombination of the data obtained, it is necessary to include the correction function in the processing algorithm.

The principle of the algorithm is as follows (Fig. 3). Data from each tube of the probe enters the common database and forms separate information lines. Subsequently, the data format software reads and decomposes each data line taking into account the displacement of the coordinates of the probe tubes relative to the measuring plane. Simultaneously, the program forms the function of the deviation of the data obtained as the difference between the atmospheric and static pressure of the measuring plane. After that, the program forms a corrective function, which is actually a set of absolute values of the deviation function. The visually given function looks like a mirror image of the surface of the deviation function. After that, a correction function is added to each row of data. As a result, we obtain a corrected series of data for each tube of the probe. Next, the software recombination module combines the corrected data of the third and fourth tube forming a matrix, which characterizes the data of the lower half of the measuring plane of the wind tunnel. In addition, since the data obtained from these tubes, in the process of their comparison, in a certain range of measurements are duplicated, it is expedient to find their mean value in this interval. In turn, each curve consists of three parts formed as a result of the recombination of data obtained from the third and fourth tube of the probe. The first part of the curve is located in the range of coordinates $X_{1}=0-60 \mathrm{~mm}$, the second part of the curve is the so-called part of the averaged data in the range $X_{2}=70-230 \mathrm{~mm}$, the third part of the curve $-\mathrm{X}_{3}=240-300 \mathrm{~mm}$. Thus, each curve characterizing the distribution of the investigated value is part of the set of curves which in the result form the general plane of the data in the coordinates $X=0-300 \mathrm{~mm}, \mathrm{Y}=0-140 \mathrm{~mm}$. A similar procedure for recombination is performed over the data obtained from the first and second tubes of the probe. As a result, an array of data for the distribution of the investigated quantity is generated which characterizes the upper half of the measuring area of the wind tunnel and is within the coordinate $X=0-300 \mathrm{~mm}, \mathrm{Y}=100-200 \mathrm{~mm}$. After that, the software recombination module combines two received mass data for the upper and lower part of the measuring plane. Averaging of duplicate data in the direction of the OY axis is parallel. As a result of this complex recombination algorithm, a common data matrix of the measured value in the coordinates $X=0-300 \mathrm{~mm}, \mathrm{Y}=0-200 \mathrm{~mm}$ is formed.

The developed algorithm allows simultaneously carrying out the corrected recombination of several data arrays for visualization of data of dynamic pressure, flow rates and standard error values. A similar procedure for recombination is performed over the data obtained from the first and second tubes of the probe. As a result, an array of data for the distribution of the investigated quantity is generated which characterizes the upper half of the measuring area of the wind tunnel and is within the coordinate $X=0-300 \mathrm{~mm}, \mathrm{Y}=100-200 \mathrm{~mm}$. After that, the software recombination module combines two received mass data for the upper and lower part of the measuring plane. Averaging of duplicate data in the direction of the OY axis is parallel. As a result of this complex recombination algorithm, a common data matrix of the measured value in the coordinates $X=0-300 \mathrm{~mm}, \mathrm{Y}=0-200 \mathrm{~mm}$ is formed.

The developed algorithm allows simultaneously carrying out the corrected recombination of several data arrays for visualization of data of dynamic pressure, flow rates and standard error values. Based on the developed algorithm in the program environment LabVIEW was created a program for graphical interpretation of the data in case of parallel decoding. In addition, the functionality of the program allows you to analyze the data obtained, depending on the type of their numerical recombination. 


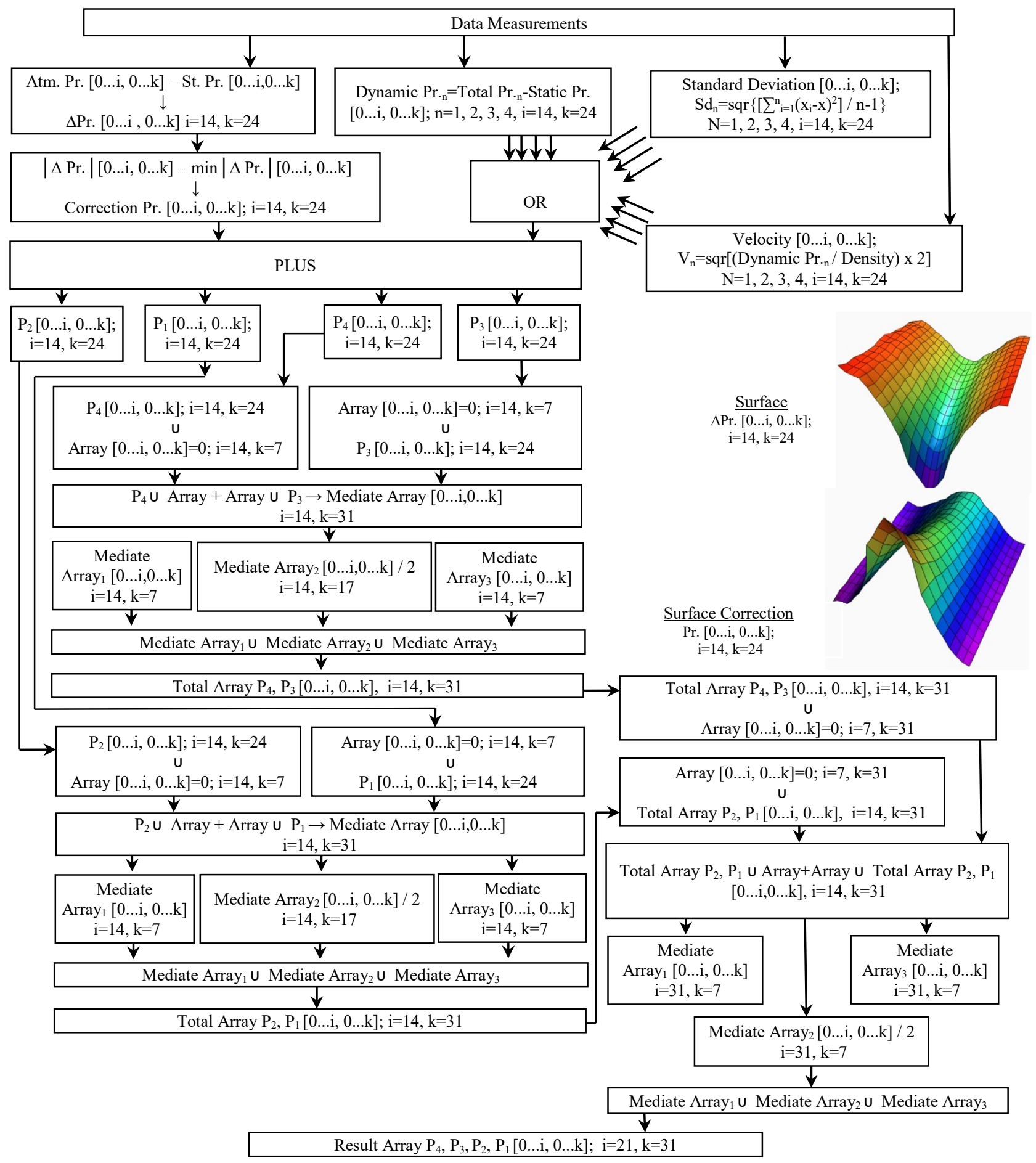

FIGURE 3. Recombination corrective algorithm of the obtained data.

Also, the program developed allows you to determine the numerical characteristics of cross-sectional sections of the obtained 3D data distribution graphs; set their maximum and minimum values and the numerical difference between the two given points of the curve; make a visual interpretation of the correction function and save the decoded mass of data. 


\section{Analysis of Experimental Data Using the Developed Algorithm}

The developed algorithm and software on its basis allows carrying out, with high precision, 3D visualization and analysis of the investigated quantities in the measuring planes of the wind tunnel. Fig. 4 shows the measurement results before and after data recombination for dynamic pressure, flow rate and standard error at $20 \mathrm{~m} \cdot \mathrm{s}^{-1}$. The analysis of this figure showed the uneven distribution of the investigated quantities (a), (c) before the corrected recombination and their uniform distribution after the corrected recombination (b), (d). At given parameters the dynamic pressure value is $220 \mathrm{~Pa}$, the value of the calculated flow velocity is $19.5 \mathrm{~m} \cdot \mathrm{s}^{-1}$.

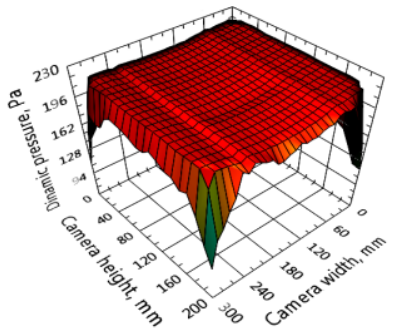

(a)

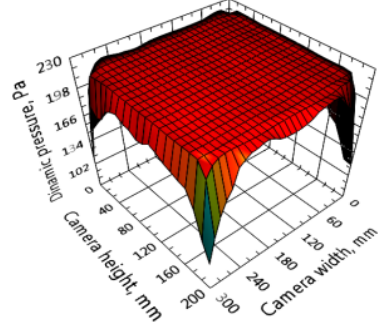

(b)

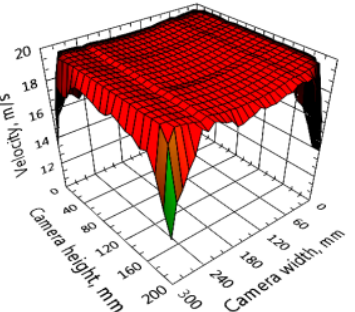

(c)

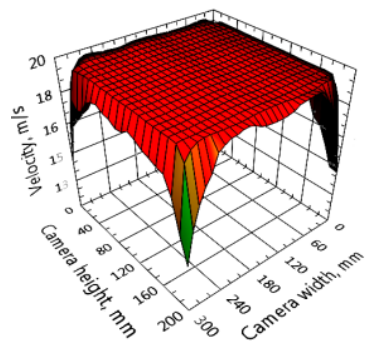

(d)

FIGURE 4. Visualization of the data obtained by the developed algorithm. (a), (c) Dynamic pressure and velocity flow before and (b), (d) after corrected recombination.

A number of experimental studies were carried out to confirm the adequacy of the proposed algorithm for corrective recombination. They were based on the measurement of dynamic pressure when placing objects that create aerodynamic resistance in the measuring section (Fig. 5). As you can see from the figure, objects have completely different geometric characteristics and different aerodynamic resistance. The specified speed of the wind flow was $20 \mathrm{~m} \cdot \mathrm{s}^{-1}$.

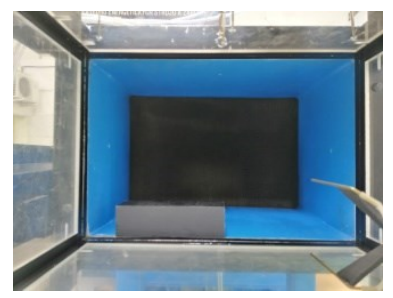

(a)

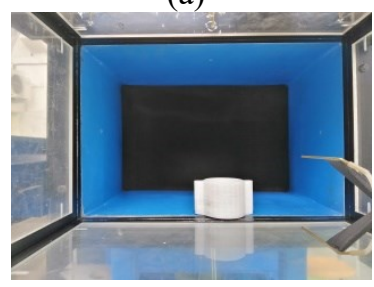

(e)

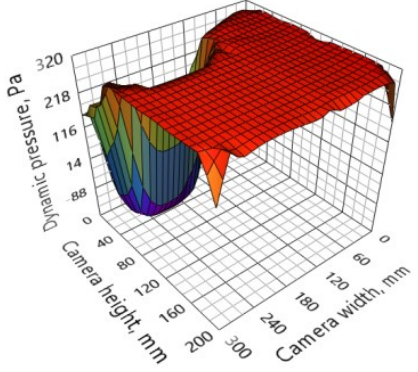

(b)

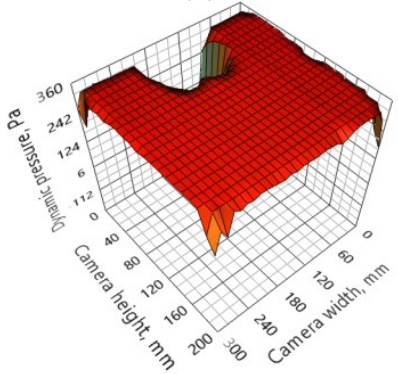

(f)

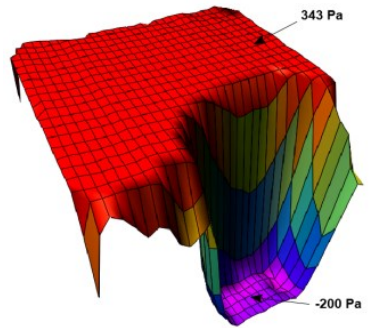

(c)

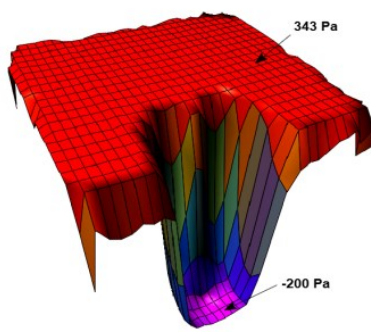

(g)

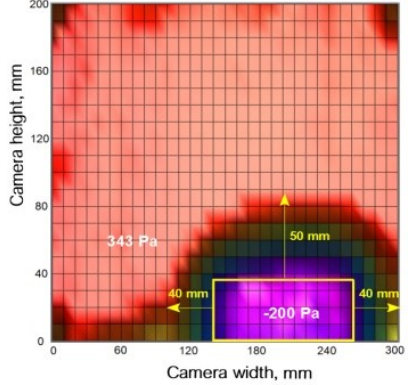

(d)

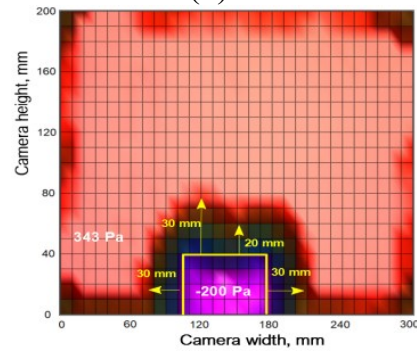

(h)

FIGURE 5. The value of the dynamic pressure in the measuring section of the wind tunnel in the presence of resistance bodies. (a) The body in the form of a rectangular parallelepiped. (e) The body with complex geometry. (b), (c), (d) The distribution of the dynamic pressure in the presence of a rectangular parallelepiped. $(\mathrm{f}),(\mathrm{g}),(\mathrm{h})$ Distribution of dynamic pressure in the presence of a complex body geometry. 
Analysis of the data showed that the dynamic pressure on stable sections of the graph is $343 \mathrm{~Pa}$. In addition, the pressure difference at the location of the bodies is up to $-200 \mathrm{~Pa}$. It is worth noting that the developed algorithm of the corrected recombination allows to visualize sharp local variations of pressure with high accuracy.

In addition, stable areas of pressure do not detect distortions in the process of processing them. A profile analysis of the resulting surfaces allowed the installation of a certain transient pressure region around the body contour. For a body in the form of a rectangular parallelepiped, this area is $40-50 \mathrm{~mm}$, while for a body with complex geometry it is $30 \mathrm{~mm}$. In addition, as can be seen on Fig. $5 \mathrm{f}, \mathrm{g}$, h, the cavity formed as a result of the pressure difference has two clearly identified rounded peaks, arising due to the generation of swirls relative to the surface of the resistance body.

The analysis of the results confirmed the adequacy of the developed algorithm of the corrected recombination of the measured data and the possibility of its further use in experimental research

\section{ACKNOWLEDGMENTS}

This work was supported by the project of Technology Agency of the Czech Republic TACR No. Th2020057 "Program Epsilon".

\section{REFERENCES}

1. J.B. Barlow, Jr. Rae, H. William and A. Pope, Low-speed wind tunnel testing (Third edition John Wiley and Sons INC, 1999), p. 713.

2. R.G. Pankhurst and D.W. Holder, Wind-tunnel technique (Sir Isaac Pitman and sons L.T.D. London, 1952), p. 702.

3. W. Nitsche and J. Szodruch, Journal of Aircraft 30, 192-200 (1993).

4. L. Cattafesta, C. Bahr and J. Mathew, "Fundamentals of wind-tunnel design", in Encyclopedia of Aerospace Engineering (John Wiley \& Sons, 2010)

5. F.M. White, Fluid mechanics (Boston McGraw-Hill, 2003), p. 885.

6. J. Colman, Wind tunnels and experimental fluid dynamics research (InTEch, 2011), p. 709.

7. G.V. Iungo, Yu-Ting $\mathrm{Wu}$ and $\mathrm{F}$. Porte, Wind tunnel aerodynamic test of six airfoils for use on small wind turbines (Colorado, Nation Renewable energy laboratory, 2004), p. 18.

8. J.D. Pereira, Wind tunnels: aerodynamics, models and experiments (Nova science publishers, Inc., 2011), p. 240.

9. G.K. Batchelor, The theory of homogeneous turbulence (Cambridge classic, Cambridge, 1953), p. 201.

10. F.K. Owen and A.K. Owen, Progress in aerospace sciences 22, 315-348 (2008).

11. N.K. Maurya, M. Maurya, A. Tyagi and Sh.P. Dwivedi, International journal of engineering and technology 7, 381-387 (2018).

12. Y. Sun, Yu. Wang, A. Da Ronch, and D. Meng, "A fast correction method of model deformation effects in wind tunnel test", Aerospace 5 (125), 19 (2018).

13. V. Uruba, P. Jonáš and O. Mazur, International Journal of Heat and Fluid Flow 28, 665-672 (2007).

14. O. Hladík and V. Uruba, Mechanical Engineering J., June 2009, $69-70$ (2009). 